\title{
EXISTENCE RESULTS FOR SEMILINEAR DIFFERENTIAL INCLUSIONS
}

\section{Zhenbin Fan AND GaNG LI}

In this paper we study the existence of mild solutions for Cauchy problem

$$
u^{\prime}(t) \in A(t) u(t)+F(t, u(t)), 0<t \leqslant T, u(0)=u_{0} .
$$

We derive conditions under which the mild solutions exist, and also get the relative compactness of the solution set, which extend and improve some existing results in this area.

\section{INTRODUCTION}

The theory of nonlinear differential inclusions in Banach spaces has been developing fast because of its extensive practical applications such as free boundary problems and moving boundary problems for partial differential equations (see $[1,20,21])$, feedback stabilisation and optimal control problems (see $[2,5,8,9,22]$ ).

In this paper we study the existence of solutions to the following semilinear evolution differential inclusion

$$
\begin{aligned}
& u^{\prime}(t) \in A(t) u(t)+F(t, u(t)), \text { almost everywhere } t, \\
& u(0)=u_{0},
\end{aligned}
$$

in a real Banach space $X$. Here $\{A(t)\}_{t \in[0, T]}$ is a family of linear operators and $F$ is a multifunction.

Different versions of this system have been investigated by many authors. Bressan [6], Kisielewicz [13], Fryszkowski [11] and Papageorgiou [18] discussed the existence results in the case when $A(t)=0$. Vrabie [23] considered the case when $A(t)=A$ generates a compact $C_{0}$-semigroup and $F(t, \cdot)$ is continuous. In [7, 12], the authors supposed that $F$ has convex and compact values and proved the existence results of (1.1), (1.2) without assuming necessarily that evolution system generated by $A(t)$ is equicontinuous, which extended the main results in $[14,19,23]$.

Received 24th January, 2007

The work is supported by PRC grant NSFC 10571150.

Copyright Clearance Centre, Inc. Serial-fee code: 0004-9727/07 \$A2.00+0.00. 
The purpose of this paper is to state that we can solve the differential inclusion (1.1), (1.2) when $F$ has nonconvex values. Techniques employed in [7, 12], have been generalised to the study of (1.1), (1.2) in this situation. That is, local existence of mild solutions is first proved, using the theory of continuous selection and a fixed point argument. Then, by careful analysis, we extend the local mild solution of (1.1), (1.2) to a maximal interval. Finally, under a stronger boundedness condition on $F$, we obtain that the set of all global solutions defined on $[0, T]$ is nonempty and relatively compact in $C(0, T ; X)$. Therefore, our work extends and improves those in $[7,12,14,19,23]$, and maybe give a way to remove the compactness for the nonlocal Cauchy problems and the periodic problems of evolution inclusions (see $[3,15,16,17,24]$ ).

The main tools in the approach followed in this work are measure of noncompactness, the theory of semilinear differential equations and multivalued analysis. A brief reminder of these is provided in Section 2. Our main results are given in Section 3.

\section{Preliminaries}

Let $Y$ and $Z$ be topological spaces, we shall use the following notations:

$$
\begin{aligned}
& P(Z)=\{A \subseteq Z: \text { nonempty }\} \\
& P_{b}(Z)=\{A \subseteq Z: \text { nonempty, bounded }\} \\
& P_{k c}(Z)=\{A \subseteq Z: \text { nonempty, compact, convex }\} .
\end{aligned}
$$

A multifunction $F: Y \rightarrow P(Z)$ is said to be upper-semicontinuous if the set $\{y \in Y ; F(y) \subseteq V\}$ is open in $Y$ for every open $V \subseteq Z ; F$ is said to be lowersemicontinuous if the set $\{y \in Y ; F(y) \cap V \neq \emptyset\}$ is open in $Y$ for every open $V \subseteq Z ; F$ is said to be closed if its graph $g r F=\{(y, z) ; z \in F(y)\}$ is closed in $Y \times Z$, that is, $\forall y \in Y$, if sequence $\left(y_{n}, z_{n}\right) \in g r F$ satisfying $\left(y_{n}, z_{n}\right) \rightarrow(y, z)$ in $Y \times Z$, we have $z \in F(y)$.

Let $(X,\|\cdot\|)$ be a real Banach Space.

We recall the following definitions.

Definition 2.1: Let $E^{+}$be the positive cone of an order Banach space $(E, \leqslant)$. A function $\Phi$ defined on the set of all bounded subsets of the Banach space $\mathrm{X}$ with values in $E^{+}$is called a measure of noncompactness on X if $\Phi(\overline{c o} \Omega)=\Phi(\Omega)$ for all bounded subsets $\Omega \subset X$, where $\overline{c o} \Omega$ stands for the closed convex hull of $\Omega$.

The measure of noncompactness $\Phi$ is said:

(i) monotone if for all bounded subsets $\Omega_{1}, \Omega_{2}$ of $X$ we have:

$$
\left(\Omega_{1} \subseteq \Omega_{2}\right) \Rightarrow\left(\Phi\left(\Omega_{1}\right) \leqslant \Phi\left(\Omega_{2}\right)\right) ;
$$

(ii) nonsingular if $\Phi(\{a\} \cup \Omega)=\Phi(\Omega)$ for every $a \in X, \Omega \in P_{b}(X)$;

(iii) regular if $\Phi(\Omega)=0$ if and only if $\Omega$ is relatively compact in $X$. 
One of the most important examples of measure of noncompactness is the noncompactness measure of Hausdorff $\chi$ defined on each bounded subset $\Omega$ of $X$ by

$$
\chi(\Omega)=\inf \{\varepsilon>0 ; \Omega \text { has a finite } \varepsilon-\text { net in } X\}
$$

It is well known that measure of noncompactness $\chi$ enjoys the above properties (i)-(iii) and other properties (see $[4,12])$.

Let $[0, T], T>0$, be a fixed interval and denote

$$
\Delta=\{(t, s) \in[0, T] \times[0, T]: 0 \leqslant s \leqslant t \leqslant T\} .
$$

We recall the following basic definition (see [21]).

Definition 2.2: A two parameter family $\{U(t, s)\}_{(t, s) \in \Delta}, U(t, s): X \rightarrow X$ bounded linear operator, $(t, s) \in \Delta$, is called an evolution system if the following conditions are satisfied:

(1) $U(s, s)=I, U(t, r) U(r, s)=U(t, s)$ for $0 \leqslant s \leqslant r \leqslant t \leqslant T$

(2) $(t, s) \rightarrow U(t, s)$ is strongly continuous on $\Delta$.

For any evolution system, we can consider the respective evolution operator $U: \Delta$ $\rightarrow L(X)$, where $L(X)$ is the space of all bounded linear operators in $X$.

We denote by $C(0, T ; X)$ the space of $X$-valued continuous functions on $[0, T]$ with the norm $\|x\|=\sup \{\|x(t)\|, t \in[0, T]\}$ and by $L^{1}(0, T ; X)$ the space of $X$-valued Bochner integrable functions on $[0, T]$ with the norm $\|f\|_{L^{1}}=\int_{0}^{T}\|f(t)\| \mathrm{d} t$.

Consider the Cauchy problem:

$$
\begin{aligned}
& u^{\prime}(t)=A(t) u(t)+h(t), \quad 0<t \leqslant T \\
& u(0)=u_{0}
\end{aligned}
$$

where $u_{0} \in X, A(t)$ satisfy the following condition:

(A) $\{A(t)\}_{t \in[0, T]}$ is a family of linear (not necessarily bounded or closed) operators, $A(t): D(A) \subset X \rightarrow X, D(A)$ not depending on $t$ and dense subset of $X$, generating an evolution operator $U: \Delta \rightarrow L(X)$ (see [21]).

Definition 2.3: A function $u \in C(0, T ; X)$ is a mild solution of (2.1), (2.2) if:

(i) $u(t)=U(t, 0) u_{0}+\int_{0}^{t} U(t, s) h(s) \mathrm{d} s, t \in[0, T]$,

(ii) $u(0)=u_{0}$.

We know that there is a unique mild solution of (2.1), (2.2) when $h \in L^{1}(0, T ; X)$. We define the mild solution operator $S$ as follows: for $h \in L^{1}(0, T ; X)$, we denote by $S(h)$ the unique mild solution of Cauchy problem (2.1), (2.2). We shall use the following conclusion (see $[7,12])$ : 
LEMma 2.1. Let $\left\{f_{n}\right\}_{n=1}^{+\infty}$ be a sequence of functions in $L^{1}(0, T ; X)$. Assume that there exist $\mu, \eta$ in $L^{1}\left(0, T ; R^{+}\right)$satisfying

$$
\sup _{n \geqslant 1}\left\|f_{n}(t)\right\| \leqslant \mu(t) \text { and } \chi\left(\left\{f_{n}(t)\right\}_{n=1}^{+\infty}\right) \leqslant \eta(t) \text { almost everywhere } t \in[0, T] .
$$

Then for all $t \in[0, T]$, we have

$$
\chi\left(\left\{S f_{n}(t)\right\}_{n=1}^{+\infty}\right) \leqslant 2 M \int_{0}^{t} \eta(s) \mathrm{d} s,
$$

where $M$ equals to $\sup _{(t, s) \in \Delta}\|U(t, s)\|_{L(X)}$.

Now, we recall some results in the theory of measurable multivalued mappings (see [22]).

The mapping $F:[0, T] \rightarrow P(X)$ is called a strongly measurable mapping if it is almost everywhere in $[0, T]$ a pointwise limit of the sequence $F_{n}:[0, T] \rightarrow P(X), n \geqslant 1$, of step mappings. Every strongly measurable multivalued mapping $F$ admits a strongly measurable selection $f:[0, T] \rightarrow X$, that is, $f$ is strongly measurable and $f(t) \in F(t)$ for almost everywhere $t \in[0, T]$.

Let $V$ be a subset of $X$.

The mapping $\Gamma:[0, T] \times X \rightarrow P(X)$ will be called a Caratheodory type of mapping on $[0, T] \times V$ if it is strongly measurable in $t$ for every $x \in V$ and continuous in $x$ for almost every $t \in[0, T]$.

The mapping $\Gamma:[0, T] \times X \rightarrow P(X)$ is integrally bounded on $[0, T] \times V$ if there exists a function $\lambda(t) \geqslant 0$ summable on $[0, T]$ such that $\|\Gamma(t, V)\|=\sup \{\|g\|: g \in F(t, x)$, $x \in V\} \leqslant \lambda(t)$ almost everywhere on $[0, T]$.

Next, we shall assume that $X$ is separable.

Let $\left(\Omega, \sum, \mu\right)$ be a measure space and $F: \Omega \rightarrow P(X)$ be closed valued. We say that $F$ is measurable if the function $\omega \rightarrow d(x, F(\omega))$ is measurable, for all $x \in X$, where $d(x, A)=\inf \{\|x-a\|: a \in A\}$. In the case when $F: \Omega \times X \rightarrow P(X)$, the measurability is understood in the sense of $\sum \times B(X)$, where $B(X)$ is the $\sigma$-algebra of Borel subsets of $X$.

We call a set $A \subset L^{1}(0, T ; X)$ decomposable if for all $u, v \in A$ and each measurable subset $I$ in $[0, T]$, we have $\mathfrak{x}_{I} u+\mathfrak{x}_{[0, T]-I} v \in A$, where $\mathfrak{x}_{I}$ stands for the characteristic function of set $I$. A multimap is decomposable if its image is a decomposable subset of $L^{1}(0, T ; X)$.

We shall use the following selection theorem (see [10]):

LeMma 2.2. Let $X$ be a separable real Banach space, $K$ a compact subset in $C(0, T ; X)$, and $G: K \rightarrow P\left(L^{1}(0, T ; X)\right)$ a nonempty, closed valued mapping which is lower-semicontinuous and decomposable. Then there exists at least one continuous function $g: K \rightarrow L^{1}(0, T ; X)$ such that $g(u) \in G(u)$, for each $u \in K$. 


\section{Main Results}

In this Section, we shall consider the following Cauchy problem for system governed by a semilinear evolution differential inclusion:

$$
\begin{aligned}
& u^{\prime}(t) \in A(t) u(t)+F(t, u(t)), \quad 0<t \leqslant T, \\
& u(0)=u_{0},
\end{aligned}
$$

where $A(t)$ satisfies $(A), F$ satisfies the following conditions:

(F1) $F:[0, T] \times X \rightarrow P(X)$ be a measurable, closed valued multifunction such that $F(t, \cdot)$ is lower-semicontinuous for almost all $t \in[0, T]$;

(F2) the mapping $F$ is integrally bounded on bounded subset of $[0, T] \times X$, that is, for every nonempty bounded set $B \subset X$, there exists a function $\omega_{B} \in L^{1}\left(0, T ; R^{+}\right)$such that for every $x \in B$,

$$
\|F(t, x)\| \leqslant \omega_{B}(t)
$$

for almost all $t \in[0, T]$;

(F3) there exists a function $k \in L^{1}\left(0, T ; R^{+}\right)$such that for every bounded $D \subset X$,

$$
\chi(F(t, D)) \leqslant k(t) \cdot \chi(D)
$$

for almost everywhere $t \in[0, T]$, where $\chi$ is the Hausdorff measure of noncompactness.

REMARK 3.1. Applying the Lemma 2.2 and using the condition (F1) and (F2), for every continuous function $x \in C(0, T ; X)$ we have an integral selection $f \in L^{1}(0, T ; X)$ of $F(\cdot, x(\cdot))$. Therefore, the set $S_{F(\cdot, x(\cdot))}^{1}$ is nonempty.

Definition 3.1: A function $u \in C(0, b ; X)(0<b \leqslant T)$ is a mild solution of (3.1), (3.2) if:

(i) $u(0)=u_{0}$,

(ii) $u(t)=U(t, 0) u_{0}+\int_{0}^{t} U(t, s) f(s) \mathrm{d} s, \quad t \in[0, b]$,

with $f \in S_{F(\cdot, u(\cdot))}^{1}$.

First, we introduce some noncompact measures. For $\Omega \subseteq C(0, T ; X)$, we define

$$
\alpha(\Omega)=\max _{E \in \Delta(\Omega)}\left(\sup _{t \in\{0, T]} \chi(E(t))\right),
$$

where $\Delta(\Omega)$ denotes the collection of all denumerable subsets of $\Omega$ and $E(t)=\{u(t)$ : $u \in E\}$. Then $\alpha$ is well defined and from the corresponding properties of Hausdorff measure of noncompactness $\chi$ it is clear that $\alpha$ is a monotone and nonsingular measure of noncompactness. 
We also define

$$
\beta(\Omega)=\max _{E \in \Delta(\Omega)}\left(\sup _{t \in[0, T]} \bmod _{C}(E(t))\right)
$$

where $\bmod _{C}(E(t))$ is the modulus of equicontinuity of the set of functions $E$ at point $t$ given by the formula

$$
\bmod _{C}(E(t))=\lim _{\delta \rightarrow 0} \sup \left\{\left\|u\left(t_{1}\right)-u\left(t_{2}\right)\right\|: t_{1}, t_{2} \in(t-\delta, t+\delta), u \in E\right\} .
$$

Then $\beta$ is well defined and also is a monotone and nonsingular measure of noncompactness. Similar definitions with $\alpha, \beta$ can be found in [8] or [12].

Now, we define

$$
H(\Omega)=\alpha(\Omega)+\beta(\Omega)
$$

Then we have the following conclusion.

LEMMA 3.1. $H$ is a monotone, nonsingular and regular measure of noncompactness defined on bounded subsets of $C(0, T ; X)$.

Proof: It is easy to check that $H$ is a monotone and nonsingular measure of noncompactness. We shall show that $H$ is regular.

Firstly, if $\Omega \subseteq C(0, T ; X)$ is relatively compact, then by the abstract version of Arzela-Ascoli Theorem, we have $H(\Omega)=0$.

On the other hand, suppose that $H(\Omega)=0$, by the definition of $H$, we obtain

$$
\alpha(\Omega)=0, \quad \beta(\Omega)=0
$$

To prove the relative compactness of $\Omega$, it remains to show that $\Omega$ is equicontinuous. Assume on the contrary, that there are $\varepsilon_{0}>0$ and sequences

$$
\left\{u_{n}\right\}_{n=1}^{+\infty} \subset \Omega,\left\{t_{n}\right\},\left\{\bar{t}_{n}\right\} \subset[0, T]
$$

such that $t_{n} \rightarrow t_{0}, \bar{t}_{n} \rightarrow t_{0}$ as $n \rightarrow+\infty$ and

$$
\left\|u_{n}\left(t_{n}\right)-u_{n}\left(\bar{t}_{n}\right)\right\| \geqslant \varepsilon_{0}
$$

for all $n \geqslant 1$. However,

$$
\left\|u_{n}\left(t_{n}\right)-u_{n}\left(\bar{t}_{n}\right)\right\| \leqslant \sup \left\{\left\|u\left(t_{n}\right)-u\left(\bar{t}_{n}\right)\right\|: u \in \Omega\right\} .
$$

Taking the upper limit, we get that

$$
\varlimsup_{n}\left\|u_{n}\left(t_{n}\right)-u_{n}\left(\bar{t}_{n}\right)\right\| \leqslant \bmod _{C}\left(\Omega\left(t_{0}\right)\right) \leqslant \beta(\Omega)=0
$$

which gives the contradiction $0<\varepsilon_{0} \leqslant 0$. Thus $\Omega \subseteq C(0, T ; X)$ is equicontinuous. This completes the proof. 
REMARK 3.2. In the proof of our main results, we shall look for some nonempty convex and compact subset $W$ of $C(0, T ; X)$, which seems to be not a simple proof. Using the above measure of noncompactness $H$, we can solve this difficulty.

Now, we give our main results in this paper.

Theorem 3.1. Let $X$ be a real separable Banach space. Assume that the conditions (A), (F1), (F2), (F3) are satisfied. Then Cauchy problem (3.1), (3.2) has at least one local mild solution.

Proof: Since the evolution operator $U$ is strongly continuous on the compact set $\Delta$, we have

$$
\|U(t, s)\|_{L(X)} \leqslant M,(t, s) \in \Delta
$$

where $M$ is defined as Lemma 2.1.

Let $r=4 M\left\|u_{0}\right\|$, we consider the closed ball

$$
B=\left\{x \in X ;\left\|x-u_{0}\right\| \leqslant r\right\} .
$$

By (3.3), we obtain

$$
\left\|[U(t, 0)-U(0,0)] u_{0}\right\| \leqslant r / 2 \text { for all } t \in(0, T],
$$

and, we can choose $h_{1} \in(0, T]$, such that

$$
M \int_{0}^{h_{1}} \omega_{B}(s) \mathrm{d} s \leqslant r / 2
$$

and

$$
q \stackrel{\text { def }}{=} 2 M \int_{0}^{h_{1}} k(s) \mathrm{d} s<1,
$$

where $\omega_{B}$ is the function from assumption (F2) and $k$ comes from (F3).

We consider also the closed set

$$
W_{0}=\left\{y \in C\left(0, h_{1} ; X\right):\left\|y(t)-u_{0}\right\| \leqslant r, \forall t \in\left[0, h_{1}\right]\right\} .
$$

Now, we define the integral multioperator

$$
\Gamma: W_{0} \rightarrow P\left(C\left(0, h_{1} ; X\right)\right)
$$

by

$$
\Gamma(x)=\left\{y \in C\left(0, h_{1} ; X\right): y(t)=U(t, 0) u_{0}+\int_{0}^{t} U(t, s) f(s) \mathrm{d} s, f \in S_{F(\cdot, x(\cdot))}^{1}\right\}
$$


It is easy to see that the fixed point of multioperator $\Gamma$ is the mild solution of (3.1), (3.2) on $\left[0, h_{1}\right]$. To prove the existence of fixed point of $\Gamma$, we shall make use of the Schauder's fixed point Theorem.

STEP 1. We claim that the multioperator $\Gamma$ maps $W_{0}$ into itself.

Let $x \in W_{0}$, then, $\left\|x(t)-u_{0}\right\| \leqslant r, \forall t \in\left[0, h_{1}\right]$, that is, $x(t) \in B, \forall t \in\left[0, h_{1}\right]$. For arbitrary $y \in \Gamma(x)$, we have that

$$
y(t)=U(t, 0) u_{0}+\int_{0}^{t} U(t, s) f(s) \mathrm{d} s, t \in\left[0, h_{1}\right]
$$

with some $f \in S_{F(\cdot, x(\cdot))}^{1}$.

So, from the hypothesis (F2) and (3.5), (3.6), it follows that

$$
\begin{aligned}
\left\|y(t)-u_{0}\right\| & \leqslant \mid U(t, 0) u_{0}-u_{0}\left\|+\int_{0}^{t}\right\| U(t, s)\|\| f(s) \| \mathrm{d} s \\
& \leqslant\left\|U(t, 0) u_{0}-u_{0}\right\|+M \int_{0}^{h} \omega_{B}(s) \mathrm{d} s \\
& \leqslant r / 2+r / 2 \\
& \leqslant r
\end{aligned}
$$

for every $t \in\left[0, h_{1}\right]$, which implies $y \in W_{0}$, that is, multioperator $\Gamma$ maps $W_{0}$ into itself. STEP 2. We show that the integral multioperator $\Gamma$ maps some nonempty convex and compact set $W \subseteq W_{0}$ into itself.

For this purpose, we first let $\left\{y_{n}\right\}_{n=1}^{+\infty} \subseteq \Gamma\left(W_{0}\right)$ be the denumerable set which achieves the maximum of $\alpha\left(\Gamma\left(W_{0}\right)\right)$, since it is a maximum.

Of course, there exists a set $\left\{x_{n}\right\}_{n=1}^{+\infty} \subseteq W_{0}$ such that $y_{n} \in \Gamma\left(x_{n}\right), n \geqslant 1$; that is,

$$
y_{n}(t)=U(t, 0) u_{0}+\int_{0}^{t} U(t, s) f_{n}(s) \mathrm{d} s=\left(S f_{n}\right)(t), t \in\left[0, h_{1}\right],
$$

with $f_{\mathfrak{n}} \in S_{F\left(\cdot, x_{n}(\cdot)\right)}^{1}$

Now, we give an estimate for $\chi\left(\left\{y_{n}(t)\right\}_{n=1}^{+\infty}\right)$. By using condition (F3), we have

$$
\chi\left(\left\{f_{n}(s)\right\}_{n=1}^{+\infty}\right) \leqslant \chi\left(F\left(s,\left\{x_{n}(s)\right\}_{n=1}^{+\infty}\right)\right) \leqslant k(s) \chi\left(\left\{x_{n}(s)\right\}_{n=1}^{+\infty}\right) .
$$

From (3.10) and Lemma 2.1, we obtain

$$
\chi\left(\left\{y_{n}(t)\right\}_{n=1}^{+\infty}\right)=\chi\left(\left\{\left(S f_{n}\right)(t)\right\}_{n=1}^{+\infty}\right) \leqslant 2 M \int_{0}^{t} k(s) \chi\left(\left\{x_{n}(s)\right\}_{n=1}^{+\infty}\right) \mathrm{d} s .
$$

From (3.7), it follows that

$$
\begin{aligned}
\sup _{t \in\left[0, h_{1}\right]} \chi\left(\left\{y_{n}(t)\right\}_{n=1}^{+\infty}\right) & \leqslant \sup _{t \in\left[0, h_{1}\right]} \chi\left(\left\{x_{n}(t)\right\}_{n=1}^{+\infty}\right) \cdot 2 M \int_{0}^{h_{1}} k(s) \mathrm{d} s \\
& \leqslant \sup _{t \in\left[0, h_{1}\right]} \chi\left(\left\{x_{n}(t)\right\}_{n=1}^{+\infty}\right) q .
\end{aligned}
$$


That is,

$$
\alpha\left(\Gamma\left(W_{0}\right)\right) \leqslant \alpha\left(W_{0}\right) q
$$

Define

$$
W_{1}=\overline{\operatorname{co}} \Gamma\left(W_{0}\right)=\overline{c o}\left\{y \in C\left(0, h_{1} ; X\right) ; y \in \Gamma(x), x \in W_{0}\right\}
$$

then it is easy to see that $W_{1}$ is a nonempty, closed, convex subset of $C\left(0, h_{1} ; X\right)$, and

$$
\begin{aligned}
W_{1}=\overline{c o} \Gamma\left(W_{0}\right) \subseteq \overline{c o} W_{0}=W_{0}, \\
\Gamma\left(W_{1}\right) \subseteq \Gamma\left(W_{0}\right) \subseteq W_{1} .
\end{aligned}
$$

As the same reason, we have that

$$
\alpha\left(\Gamma\left(W_{1}\right)\right) \leqslant \alpha\left(W_{1}\right) q \leqslant \alpha\left(W_{0}\right) q^{2}
$$

Define

$$
W_{2}=\overline{c o} \Gamma\left(W_{1}\right)=\overline{c o}\left\{y \in C\left(0, h_{1} ; X\right) ; y \in \Gamma(x), x \in W_{1}\right\},
$$

then we have that $W_{2}$ is a nonempty, closed, convex subset of $C\left(0, h_{1} ; X\right)$, and

$$
\begin{gathered}
W_{2} \subseteq W_{1} \subseteq W_{0} \\
\alpha\left(\Gamma\left(W_{2}\right)\right) \leqslant \alpha\left(W_{0}\right) q^{3}
\end{gathered}
$$

Continuing this way, we get a decreasing sequence $\left\{W_{n}\right\}_{n=0}^{+\infty}$, which are nonempty, closed, convex, bounded subsets of $C\left(0, h_{1} ; X\right)$, and

$$
\alpha\left(\Gamma\left(W_{n}\right)\right) \leqslant \alpha\left(W_{0}\right) q^{n+1}
$$

which implies that $\alpha\left(W_{n}\right) \rightarrow 0$, as $n \rightarrow+\infty$.

Coming back to the definition of $\alpha$, we obtain that

$$
\chi\left(E_{n}(t)\right) \rightarrow 0, \quad n \rightarrow+\infty
$$

uniformly in $t \in\left[0, h_{1}\right]$ with any countable subset $E_{n} \subseteq W_{n}$.

Now, we consider $\beta\left(W_{n}\right)$. Suppose that the sequence $\left\{y_{m}^{(n)}\right\}_{m=1}^{+\infty} \subset \Gamma\left(W_{n-1}\right)$ achieves the maximum of $\alpha\left(W_{n}\right)$ and $\beta\left(W_{n}\right)$. From (3.14), it follows that

$$
\varepsilon_{n} \triangleq \chi\left(\left\{y_{m}^{(n)}(t)\right\}_{m=1}^{+\infty}\right) \rightarrow 0, n \rightarrow+\infty,
$$

uniformly in $t \in\left[0, h_{1}\right]$.

For fixed $t_{0} \in\left(0, h_{1}\right)$, we take $\delta_{0}, 0<\delta_{0}<\min \left\{t_{0}, h_{1}-t_{0}\right\}$ such that

$$
\int_{t_{0}-\delta_{0}}^{t_{0}+\delta_{0}}\|f(s)\| \mathrm{d} s \leqslant \varepsilon_{n}, \quad \forall f(s) \in \omega_{B}(s),
$$


where $B$ comes from (3.4).

Further, from (3.15) and the definition of $\chi$, there exist $v_{i} \in X, 1 \leqslant i \leqslant k$, such that

$$
\left\{y_{m}^{(n)}\left(t_{0}-\delta_{0}\right)\right\}_{m=1}^{+\infty} \subset \bigcup_{i=1}^{k} B\left(v_{i}, 2 \varepsilon_{n}\right)
$$

And, for each $y \in\left\{y_{m}^{(n)}\right\}_{m=1}^{+\infty}$, there exists $x \in W_{n-1}$ such that

$$
\begin{aligned}
& y\left(t_{1}\right)=U\left(t_{1}, t_{0}-\delta_{0}\right) y\left(t_{0}-\delta_{0}\right)+\int_{t_{0}-\delta_{0}}^{t_{1}} U\left(t_{1}, s\right) f(s) \mathrm{d} s, \\
& y\left(t_{2}\right)=U\left(t_{2}, t_{0}-\delta_{0}\right) y\left(t_{0}-\delta_{0}\right)+\int_{t_{0}-\delta_{0}}^{t_{2}} U\left(t_{2}, s\right) f(s) \mathrm{d} s
\end{aligned}
$$

for $t_{1}, t_{2} \in\left(t_{0}-\delta_{0}, t_{0}+\delta_{0}\right)$, with $f \in S_{F(\cdot, x(\cdot))}^{1} \cdot$

Now, we give an estimate for $\bmod _{C}\left(\left\{y_{m}^{(n)}\right\}_{m=1}^{+\infty}\left(t_{0}\right)\right)$. By the strongly continuity of evolution operator $U$, there exists $\delta, 0<\delta<\delta_{0}$, such that

$$
\left\|U\left(t_{1}, t_{0}-\delta_{0}\right) v_{i}-U\left(t_{2}, t_{0}-\delta_{0}\right) v_{i}\right\| \leqslant \varepsilon_{n}, \quad i=1,2, \cdots, k
$$

for $t_{1}, t_{2} \in\left(t_{0}-\delta, t_{0}+\delta\right)$. So, from (3.16)-(3.20), we have

$$
\begin{aligned}
\left\|y\left(t_{1}\right)-y\left(t_{2}\right)\right\| \leqslant \| U\left(t_{1}, t_{0}\right. & \left.-\delta_{0}\right) y\left(t_{0}-\delta_{0}\right)-U\left(t_{1}, t_{0}-\delta_{0}\right) v_{i} \| \\
& +\left\|U\left(t_{2}, t_{0}-\delta_{0}\right) y\left(t_{0}-\delta_{0}\right)-U\left(t_{2}, t_{0}-\delta_{0}\right) v_{i}\right\| \\
& +\left\|U\left(t_{1}, t_{0}-\delta_{0}\right) v_{i}-U\left(t_{2}, t_{0}-\delta_{0}\right) v_{i}\right\|+2 M \varepsilon_{n}
\end{aligned}
$$

for all $y \in\left\{y_{m}^{(\mathfrak{n})}\right\}_{m=1}^{+\infty}$ and $t_{1}, t_{2} \in\left(t_{0}-\delta, t_{0}+\delta\right)$. That is,

$$
\bmod _{C}\left(\left\{y_{m}^{(n)}\right\}_{m=1}^{+\infty}\left(t_{0}\right)\right) \leqslant(6 M+1) \varepsilon_{n} .
$$

For $t_{0}=0$ or $h_{1}$, we can also verify the above inequality. Thus, from the definition of $H$, it follows that

$$
H\left(W_{n}\right) \leqslant(6 M+2) \varepsilon_{n} \rightarrow 0, \quad n \rightarrow+\infty .
$$

Therefore, the set $W=\cap_{n \geqslant 0} W_{n}$ is a nonempty, convex, compact subset of $C\left(0, h_{1} ; X\right)$, since $H$ is a monotone, nonsingular and regular measure of noncompactness (see Lemma 3.1 and [4]). Moreover, $\Gamma$ maps $W$ into itself.

STEP 3. We claim that Cauchy problem (3.1), (3.2) has a local mild solution.

Let $G: W \rightarrow P\left(L^{1}\left(0, h_{1} ; X\right)\right)$ defined by

$$
G(u)=S_{F(\cdot, u(\cdot))}^{1}=\left\{f \in L^{1}\left(0, h_{1} ; X\right): f(t) \in F(t, u(t)), \text { almost all } t\right\} .
$$


Then it is easy to show that $G$ is lower-semicontinuous and has closed decomposable values due to the condition (F1). Thus, by Lemma 2.2 , there is a continuous function $g: W \rightarrow L^{1}\left(0, h_{1} ; X\right)$ such that $g(u) \in S_{F(\cdot, u(\cdot))}^{1}$ for every $u \in W$.

So $S \circ g: W \rightarrow W$ is a single valued mapping and $(S \circ g)(x) \in \Gamma(x)$ for every $x \in W$, where $S$ be the mild solution operator with initial value $u_{0}$.

Moreover, $S: L^{1}\left(0, h_{1} ; X\right) \rightarrow C\left(0, h_{1} ; X\right)$ also is a continuous mapping. Hence $S \circ g: W \rightarrow W$ is continuous.

Therefore, there exists at least one fixed point $u_{1} \in C\left(0, h_{1} ; X\right)$ of $S \circ g$ due to the Schauder's fixed point Theorem, that is,

$$
u_{1}(t)=U(t, 0) u_{0}+\int_{0}^{t} U(t, s) f_{1}(s) \mathrm{d} s
$$

for every $t \in\left[0, h_{1}\right]$ and almost everywhere $s \in[0, t]$, with $f_{1}(s) \in F\left(s, u_{1}(s)\right)$, which is the local mild solution of (3.1), (3.2). This completes the proof.

Now, we can extend the local mild solution of (3.1), (3.2) to a maximal interval.

THEOREM 3.2. Assume that the conditions of Theorem 3.1 are satisfied. Then there exists a mild solution $u$ of (3.1), (3.2) defined on a maximal interval of existence $[0, T]$ or $\left[0, T_{0}\right), T_{0} \leqslant T$. In the second case, the solution $u$ is unbounded on $\left[0, T_{0}\right)$.

Proof: On account of Theorem 3.1, we get a local mild solution $u_{1} \in C\left(0, h_{1} ; X\right)$ of (3.1), (3.2). So We can consider the following Cauchy problem

$$
\begin{aligned}
u^{\prime}(t) & \in A(t) u(t)+F(t, u(t)), \quad h_{1}<t \leqslant T, \\
u\left(h_{1}\right) & =u_{1}\left(h_{1}\right) .
\end{aligned}
$$

Similarly, we can get a local mild solution $u_{2} \in C\left(h_{1}, h_{2} ; X\right)$, that is,

$$
u_{2}(t)=U\left(t, h_{1}\right) u_{1}\left(h_{1}\right)+\int_{h_{1}}^{t} U(t, s) f_{2}(s) \mathrm{d} s
$$

for $t \in\left[h_{1}, h_{2}\right]$ and almost everywhere $s \in\left[h_{1}, t\right]$, with $f_{2}(s) \in F\left(s, u_{2}(s)\right)$.

Let

$$
\begin{aligned}
u^{(1)}(t) & =u_{1}(t), \quad \forall t \in\left[0, h_{1}\right] ; \\
u^{(2)}(t) & = \begin{cases}u^{(1)}(t), & t \in\left[0, h_{1}\right], \\
u_{2}(t), & t \in\left(h_{1}, h_{2}\right] .\end{cases}
\end{aligned}
$$

Hence, from (3.21) and (3.22), we get that

$$
u^{(2)}(t)=U(t, 0) u_{0}+\int_{0}^{t} U(t, s) f^{(2)}(s) \mathrm{d} s
$$


for each $t \in\left[0, h_{2}\right]$, with

$$
f^{(2)}=f_{1} æ_{\left[0, t_{1}\right]}+f_{2} æ_{\left(t_{1}, t_{2}\right]} \in S_{F\left(\cdot, u^{(2)}(\cdot)\right)}^{1},
$$

which implies that $u^{(2)}$ is a mild solution of $(3.1),(3.2)$ on $\left[0, h_{2}\right]$.

We denote by $\mathfrak{J}$ a collection of all continuous functions $u$ defined on $\left[0, h^{u}\right]$, $0<h^{u} \leqslant T$, with values in $X$ such that $u$ is a mild solution of (3.1), (3.2). Clearly, the set $\mathfrak{J}$ is nonempty. We introduce a partial order $\preceq$ on $\mathfrak{J}$ in the following way: the relation $u^{(1)} \preceq u^{(2)}$ means that $h^{(1)} \leqslant h^{(2)}$ and $f^{(1)}(t)=f^{(2)}(t)$ for $t \in\left[0, h^{(1)}\right]$, with

$$
f^{(1)} \in S_{F\left(\cdot, u^{(1)}(\cdot)\right)}^{1}, f^{(2)} \in S_{F\left(\cdot, u^{(2)}(\cdot)\right)}^{1} .
$$

Let $\left(u^{(\alpha)}(\cdot)\right)_{\alpha}$ be a chain in $(\mathfrak{J}, \preceq)$. Take $h_{0}=\sup _{\alpha} h^{(\alpha)}$. For every $t \in\left[0, h_{0}\right)$, we define the function

$$
\bar{u}(t)=U(t, 0) u_{0}+\int_{0}^{t} U(t, s) f(s) \mathrm{d} s,
$$

where $f(s) \in F\left(s, u^{(\alpha)}(s)\right)$ for some $\alpha$ and almost everywhere $s \in[0, t]$. Then, it is easy to check that $u^{(\alpha)} \preceq \bar{u}$ for every $u^{(\alpha)}$ in the chain. That is, $\bar{u}$ is the upper bound of the chain. Thus, according to Zorn's lemma there exists a mild solution $u^{*}$ of (3.1), (3.2) defined on a maximal interval of existence $[0, T]$ or $\left[0, T_{0}\right)$ with $T_{0} \leqslant T$. Suppose now that $u^{*}$ is defined on $\left[0, T_{0}\right)$ and is bounded. Then we can see that $u^{*}$ is a mild solution on every interval $[0, b]$ for $0<b<T_{0}$.

Now, for each $\varepsilon>0$, there exists $\delta_{1}>0$ such that

$$
u^{*}(t)=U\left(t, T_{0}-\delta_{1}\right) u^{*}\left(T_{0}-\delta_{1}\right)+\int_{T_{0}-\delta_{1}}^{t} U(t, s) f(s) \mathrm{d} s
$$

for $t \in\left[T_{0}-\delta_{1}, T_{0}\right)$ and

$$
\int_{T_{0}-\delta_{1}}^{T_{0}}\|f(s)\| \mathrm{d} s \leqslant \varepsilon
$$

with $f \in S_{F\left(, u^{*}(\cdot)\right)}^{1}$ (noting that we have used the boundedness of $u^{*}$ here).

Let $\left\{t_{n}\right\}_{n=1}^{+\infty}$ be a nondecreasing sequence such that $t_{n} \rightarrow T_{0}$, as $n \rightarrow+\infty$. Then there exist $\delta_{2}, 0<\delta_{2}<\delta_{1}$ and $N>0$ such that

$$
\left\|U\left(t_{n}, T_{0}-\delta_{1}\right) u^{*}\left(T_{0}-\delta_{1}\right)-U\left(t_{m}, T_{0}-\delta_{1}\right) u^{*}\left(T_{0}-\delta_{1}\right)\right\| \leqslant \varepsilon
$$

for all $m, n>N,\left|t_{n}-T_{0}\right| \leqslant \delta_{2},\left|t_{m}-T_{0}\right| \leqslant \delta_{2}$. So, from (3.23)-(3.25), it follows that

$$
\left\|u^{*}\left(t_{n}\right)-u^{*}\left(t_{m}\right)\right\| \leqslant(2 M+1) \varepsilon .
$$

Therefore, the sequence $\left\{u^{*}\left(t_{n}\right)\right\}_{n=1}^{+\infty}$ is a Cauchy sequence and the limit of $u^{*}(t)$ when $t \rightarrow T_{0}$ exists, contrary to the maximality of the interval $\left[0, T_{0}\right)$. This completes the proof. 
REMARK 3.3. In the Step 2 of the proof of Theorem 3.1, we do not use the measure of noncompactness $\alpha$ to get the compact set $W$ directly, because $\alpha$ is not a regular measure of noncompactness. However, we can calculate the modulus of equicontinuity of $W_{n}$ at every point $t \in\left[0, h_{1}\right]$ through the Hausdorff measure of noncompactness of the section of $W_{n}$. So we define the regular measure of noncompactness $H$ which makes us to obtain the compact set $W$.

REMARK 3.4. The results of above two Theorems extend and improve those in $[5,6$, $9,11,13,18$, where $A(t)=0$.

Finally, under a stronger boundedness condition on $F$, we obtain the global existence result.

Corollary 3.1. Let $X$ be a real separable Banach space. Assume that the conditions (A), (F1), (F3) are satisfied, and that

(F2)' there exists a function $\eta \in L^{1}(0, T ; X)$ such that for every $x \in X$ we have

$$
\|F(t, x)\| \leqslant \eta(t)(1+\|x\|) \quad \text { almost all } t \in[0, T] .
$$

Then the mild solutions of (3.1), (3.2) on the whole $[0, T]$ is a nonempty and relatively compact subset of the space $C(0, T ; X)$.

Proof: If we suppose that $u$ is a mild solution of (3.1), (3.2) defined on $[0, T]$, then we have

$$
u(t)=U(t, 0) u_{0}+\int_{0}^{t} U(t, s) f(s) \mathrm{d} s, t \in[0, T],
$$

with $f \in S_{F(\cdot, u(\cdot))}^{1}$. From (F2)', it follows that

$$
\|u(t)\| \leqslant M\left\|u_{0}\right\|+M\|\eta\|_{L^{1}}+M \int_{0}^{t} \eta(s)\|u(s)\| \mathrm{d} s .
$$

By using the Gronwall inequality, we get that

$$
\|u(t)\| \leqslant M\left(\left\|u_{0}\right\|+\|\eta\|_{L^{1}}\right) e^{M \int_{0}^{t} \eta(\tau) \mathrm{d} \tau},
$$

that is, $u$ is bounded on the whole interval $[0, T]$, which implies that the mild solution of (3.1), (3.2) on the whole interval $[0, T]$ is nonempty due to the Theorem 3.2. Moreover, from the proof of Theorem 3.1, we can see that every local solution of (3.1), (3.2) belongs to a compact set. So, every section of solution set on the whole interval $[0, T]$ is relatively compact, and the modulus of equicontinuity of which at every point $t$ equals zero. Therefore, on account of the proof of Lemma 3.1, the mild solutions of (3.1), (3.2) on the whole interval $[0, T]$ is relatively compact subset of the space $C(0, T ; X)$. This completes the proof. 
REMARK 3.5. Our existence results of solutions to differential inclusion do not need the compactness of the evolution system $\mathrm{U}(\mathrm{t}, \mathrm{s})$, even its equicontinuity. Moreover, we get the maximal interval of existence of mild solutions in the case when $F$ satisfies a weaker growth condition (F2) and the relative compactness of the set of all global mild solutions when $F$ satisfies a stronger boundedness condition (F2)'. Therefore, our work extends and improves those in $[7,12,14,19,23]$, and also gives a way to remove the compactness for the nonlocal initial problem and the periodic problem of evolution inclusions in Banach space.

\section{REFERENCES}

[1] J.P. Aubin and A. Cellina, Differential inclusions, Grundlehren der Mathematischen Wissenschaften 264 (Springer-Verlag, Berlin, 1984).

[2] N.U. Ahmed, Optimization and indentifilation of systems governed by evolution equations in Banach spaces, Pitman Research Notes in Mathematics 184 (Longman, London, 1988).

[3] H. Amann, 'Periodic solutions of semi-linear parabolic equations', in Nonlinear Analysis, A Collection of Papers in Honor of Erich Roth (Academic Press, New York, 1978), pp. 1-29.

[4] J. Banas and K. Goebel, Measure of noncompactness in Banach spaces, Lecture Notes in Pure and Appl. Math. 60 (Marcel Dekker, New York, 1980).

[5] F.S. De Blasi and G. Pianigiani, 'Nonconvex-valued differential inclusions in Banach spaces', J. Math. Anal. Appl. 157 (1991), 469-494.

[6] A. Bressan, 'On differential relations with lower semicontinuous right hand side', J. Differential Equations 37 (1980), 89-97.

[7] T. Cardinali and P. Rubbioni, 'On the existence of mild solutions of semilinear evolution differential inclusions', J. Math. Anal. Appl. 308 (2005), 620-635.

[8] J.F. Couchouron and M. Kamenski, 'Differential inclusions and optimal control', Lecture Notes in Nonlinear Anal. 2 (1998).

[9] H. Frankowska, 'A priori estimate for operational differential inclusions', J. Differential Equations 84 (1990), 100-128.

[10] A. Fryszkowski, 'Continuous selections for a class of nonconvex multivalued maps', Studia Math. 76 (1983), 163-174.

[11] A. Fryszkowiski, 'Existence of solution of functional differential inclusions in nonconvex case', Ann. Polon. Math. 45 (1985), 121-124.

[12] M. Kamenskii, V. Obukhovskii and P. Zecca, Condensing multivalued maps and semilinear differential inclusions in Banach spaces, De Gruyter Ser. Nonlinear Anal. Appl. 7 (de Gruyter, Berlin, 2001).

[13] M. Kisielewicz, 'Multivalued differential equations in separable Banach spaces', J. Optim. Theory Appl. 37 (231-249).

[14] Y. Li, 'The global solutions of initial value problem for abstract semilinear evolution equation', Acta Anal. Funct. Appl. 3 (2001), 339-347.

[15] J. Liang, J. Van Casteren and T.-J. Xiao, 'Nonlocal Cauchy problems for semilinear evolution equations', Nonlinear Anal. Ser. A 50 (2002), 173-189. 
[16] J. Liang, J. Liu and T.-J. Xiao, 'Nonlocal Cauchy problems governed by compact operator families', Nonlinear Anal. 57 (2004), 183-189.

[17] J. Liu, T. Naito and N.V. Minh, 'Bounded and periodic solutions of infinite delay evolution equations', J. Math. Anal. Appl. 286 (2003), 705-712.

[18] N.S. Papageougiou, 'Functional-differential inclusions in Banach spaces with nonconvex right hand side', Funkcial Ekvac. 32 (1989), 145-156.

[19] N.S. Papageorgiou, 'On integral inclusions of Volterra type in Banach spaces', Czechoslovak Math. J. 42 (1992), 693-714.

[20] N.H. Pavel, 'Nolinear evolution equations and semiguoups', Lecture Notes in Math. 1260 (Springer-Verlag, Berlin).

[21] A. Pazy, Semigroups of linear operators and applications to partial differential equations (Springer-Verlag, New York, 1983).

[22] A. Tolstonogov, Differential inclusions in a Banach space, Mathematics and its Applications 524 (Kluwer Academic Publishers, Dordrecht, 2000).

[23] I.I. Vrabie, 'Some compactness methods in the theory of nonlinear evolution equations with applications to partial differential equations', in Partial Differential Equations, Banach Center Publ. 19 (Pwn-Polish Scientific Publ., Warasw, 1987), pp. 351-361.

[24] X. Xue, 'Nonlinear differential equations with nonlocal conditions in Banach spaces', Nonlinear Anal. 63 (2005), 575-586.

Department of Mathematics

Yangzhou University

Yangzhou, Jiangsu 225002

Peoples Republic of China

and

Yangzhou Polytechnic College

Yangzhou, Jiangsu 225002

Peoples Republic of China

e-mail: fzbmath@yahoo.com.cn
Department of Mathematics

Yangzhou University

Yangzhou, Jiangsu 225002

Peoples Republic of China 